\title{
Method of 3D Solar Energy Conversion
}

\author{
Valeri I. Kotelnikov and Elena A. Ryazanova \\ Tuvinian Research Institute SB RAS, International st., 117 A, Kyzyl 667007, Republic of Tuva, Russia
}

\begin{abstract}
The problem of collecting solar energy and increasing its efficiency was studied in this paper. It was discovered that a 3DPV (three-dimensional photovoltaic) structures can generate greater amounts of measured energy densities than stationary flat PV panels (rate: 2 to 20). It has been found that the same structures work better not only because they are made in 3D but because PV panels do not have linear dependency on geometry. It seems that the conversion efficiency depends on the process of absorption of the solar energy, too, or in other words on the E. Yablonovich limit. The findings suggest that the quantity of material of solar panels may be reduced to generate the same amount of electricity.
\end{abstract}

Key words: Solar energy, efficiency, photovoltaic technology, electricity generation, energy transmission.

\section{Introduction}

The Sun is the ultimate source of energy that sustains life. In one hour the Sun exposes the Earth with enough solar energy to provide power for the entire world for one year. If a small portion of this energy is collected and cost-effectively converted into electricity, the Earth can be inhabited for as long as the Sun exists without worrying about possible shortage of energy. This single fact has propelled the solar industry on the path of solid growth.

Converting the solar stream into affordable electricity is a challenge. The main issues on the way to free usage of PV technology are: system price (3-5 $\$ /$ Watt-peak) $\sim 60 \%$ of which is installation price and limited number of peak sun-hours in most locations.

The main approach applied so far to resolve these issues was to search for low-cost active layers with higher efficiency of power conversion. However, efficiency level increase can only partially reduce the installation cost and cannot change the way of solar energy generation since this aspect is related to the PV system design peculiarities.

Bernardi et al. [1] do a research about the problem of collecting solar energy in three dimensions.

Corresponding author: Valeri I. Kotelnikov, Ph.D., research field: alternative nontraditional energy.
Authors demonstrate that absorbers and reflectors can be combined in the conditions of absence of sun tracking to build 3DPV (three-dimensional photovoltaic) structures that can generate measured energy densities (energy per base area, $\mathrm{kWh} / \mathrm{m}^{2}$ ) in greater amounts than stationary flat PV panels (rate: 2 to 20). The structures considered in the study were compared to the flat panels with dual-axis sun tracking (rate: 1.3 to 1.8). The increased energy density is countered by a larger solar cell area per generated energy for 3DPV compared to flat panels (rate: 1.5 to 4) (from original text [1]), but shows a vast range of improvements. Authors state that 3DPV can double the number of peak power generation hours and dramatically reduce the seasonal and weather variations of solar energy generation compared to flat panel design.

Yiling et al. [2] demonstrates a new light trapping technique that exploits dielectric core-shell optical antennas to enhance solar absorption. This approach can allow the thickness of active materials in solar cells lowered by almost 1 order the magnitude without damaging solar absorption capability (from original text [2]).

Authors study the fundamental mechanism of the enhancement and demonstrates that the size of the semiconductor and the dielectric parts in the core-shell structure are key ones for optimizing the enhancement. 


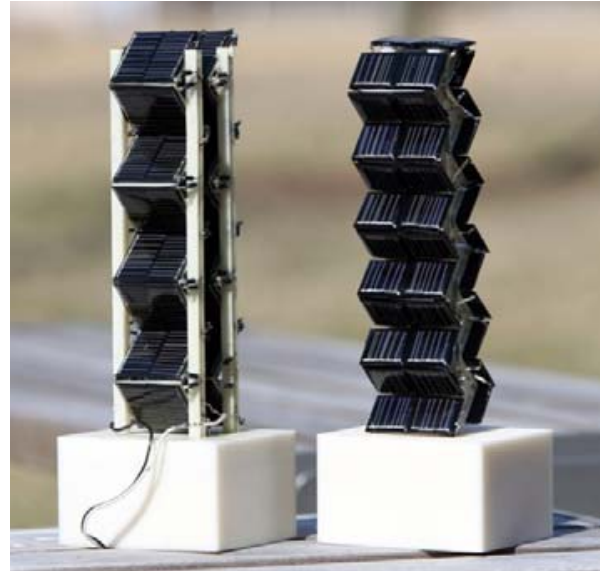

Fig. 1 Three-dimensional photovoltaic structure.

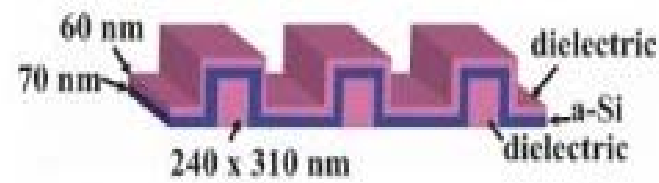

Fig. 2 Dielectric core-shell optical antennas.

Mavrokefalos et al. [3] show that thin-film crystalline silicon (c-Si) solar cells with light-trapping structures can enhance light absorption within the semiconductor absorber layer and reduce material usage. Authors demonstrate that the inverted nanopyramid light-trapping scheme for c-Si thin films fabricated at wafer scale by low-cost wet etching process significantly enhances absorption within the c-Si layer. A broadband enhancement of absorption that approaches the Yablonovitch limit [4] is achieved with the minimal angle dependence.

It was calculated that the surface of nanopyramid (Sp) and surface of the part of hatching (Sl) with same linear dimensions are equal.

$$
\mathrm{Sp}=\mathrm{p}^{*} \mathrm{a} / 2
$$

where,

$\mathrm{p}$-perimeter

a-apothem

$$
\mathrm{Sl}=2 * \mathrm{a} * \mathrm{l}
$$

where,

$$
\begin{aligned}
& \text { a-apothem } \\
& \mathrm{l} \text {-length } \\
& \mathrm{l}=\mathrm{p} / 4
\end{aligned}
$$

$\mathrm{l}$ and $\mathrm{p} / 4$ are replaced:

$$
\mathrm{Sl}=2 * \mathrm{a} * \mathrm{p} / 4=\mathrm{p} * \mathrm{a} / 2=\mathrm{Sp}
$$

It has been proven that $\mathrm{Sp}$ and $\mathrm{Sl}$ are equal. Fabricating nanopyramides on the solar cell surface is a complex and expensive process. Still scratched solar cells with higher efficiency could be manufactured.

To sum up the statements mentioned above it can be concluded that a way of improving solar cells efficiency is found. It is more efficient to use the ratio of visible part and whole square of solar cell. In accordance to this idea, a new angular PV converter can be built.

\section{Experimental Results}

It is a device with at least two solar cells placed opposite to each other with its wider part directed to the sun. It has two pairs of solar cells one (external) positioned horizontally and the other (internal) positioned at the angle between cells.
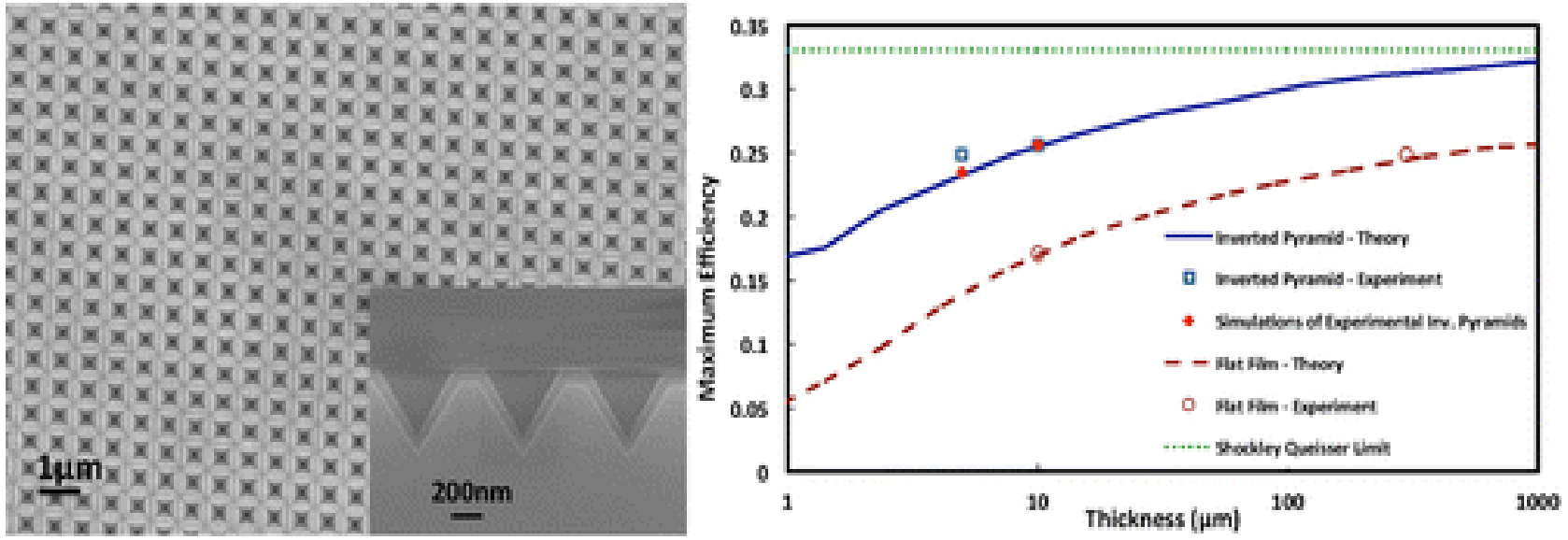

Fig. 3 Inverted nanopyramid light-trapping scheme for c-Si thin films. 


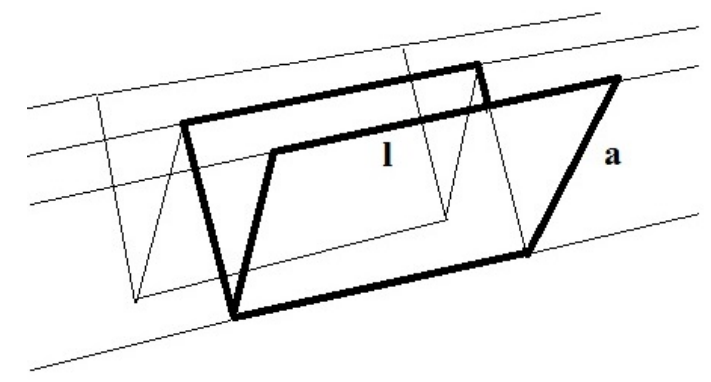

Fig. 4 Flat cell texture.

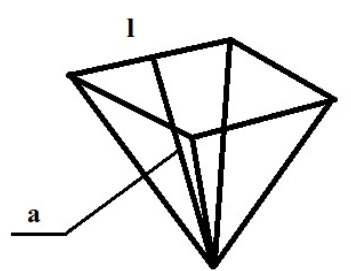

Fig. 5 Inverted nanopyramid.

Table 1 Dependence power on geometry.

\begin{tabular}{ll}
\hline Geometry & Power Watts \\
\hline Horizontally positioned & 0.19 \\
Angle between cells & 0.40 \\
\hline
\end{tabular}

The device test results are presented in Table 1. Solar cell dimensions: length $-150 \mathrm{~mm}$, width $-80 \mathrm{~mm}$.

Dependence of power of solar cells and their geometry is presented below, Fig. 6. Analysis of the results shows non-linear dependence of photons absorption.

Power of solar cell was doubled. It can be used with a wide range of inorganic and organic active materials. 3D solar cells that collect almost all of the light received, could boost the efficiency of photovoltaic systems while reducing their size, weight and mechanical complexity.

\section{Conclusions}

Probably the efficiency of PV conversion depends on the ratio of visible part and whole square of solar cell. The data demonstrate that focline is more efficient than the inverted nanopyramid light trapping. The focline can be fabricated at any stage; it enhances

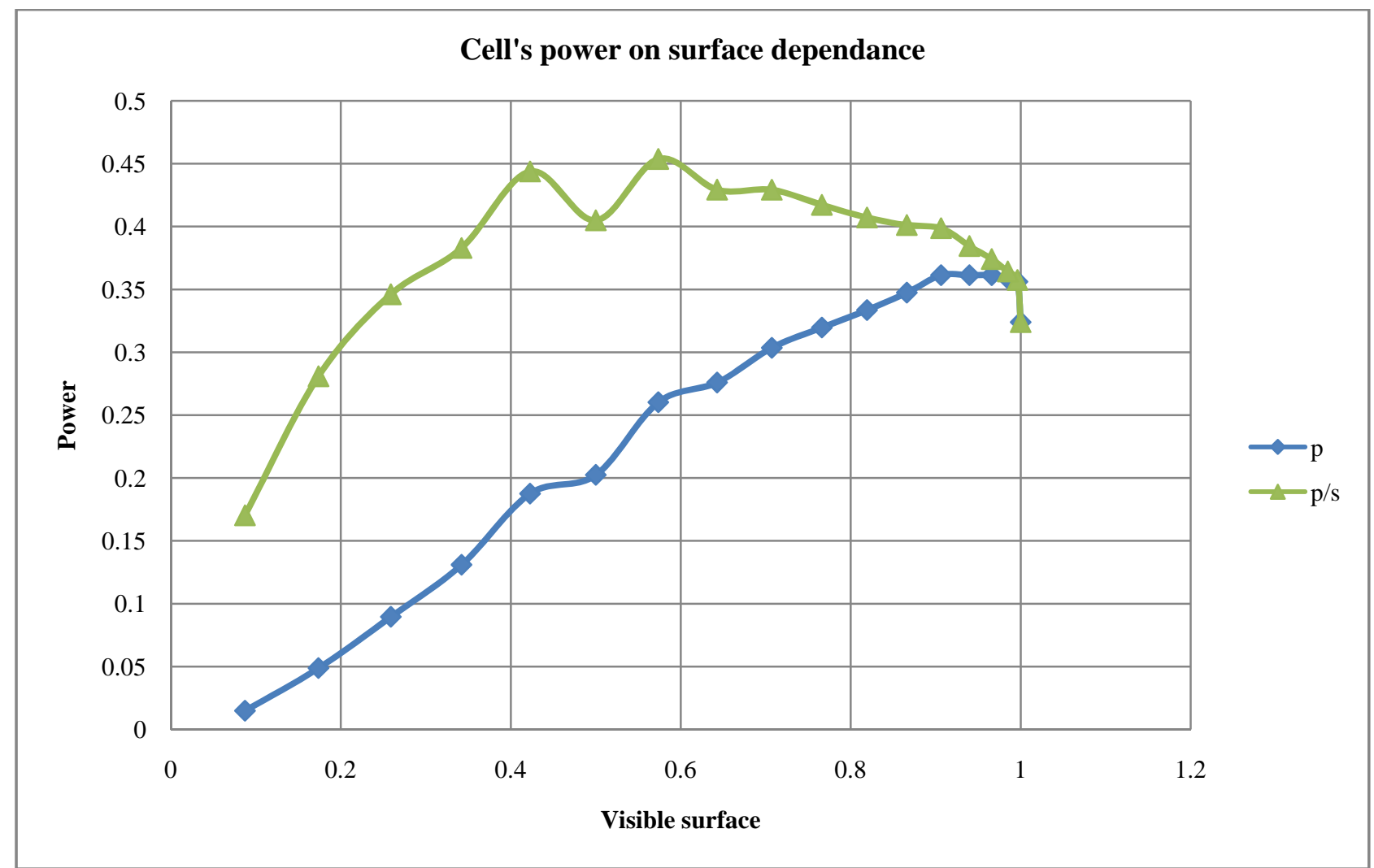

Fig. 6 Power productivity of solar cell.

p-power of cell, $\mathrm{p} / \mathrm{s}$ - power per area of lighting. 
absorption for any type of solar cell. It is the cheapest way to improve the solar cells technology.

Where can it be used? In the PV industry, of course, but also in other fields, heat radiation (infrared) from fuel combustion can be converted directly to electricity. It is estimated that somewhere between 20 to $50 \%$ of industrial energy input is lost as waste in form of hot exhausts, cooling water, or heat from hot equipment surfaces and heated products. Typically, during the process the latent energy from fuel stock (coal, gas, uranium) is converted into mechanical energy and ultimately into electrical energy by a generator. In all of these cases, though, some of the input energy is lost during the process.

The generation efficiency varies widely depending on the technology used. In a traditional coal plant, for example, only about $30-35 \%$ of the energy ends up as electricity. There is, therefore, a tremendous economic and ecological incentive to improve the efficiency of power generation so that greater quantity of input fuel turns into the output electricity.

The transmission and distribution system comprises everything between generation plant and end-use location. During the process some of the energy produced by the generator is lost because of the wires and equipment resistance. Most of this energy is converted to heat. The quantity of energy which can be lost during the process of its production depends greatly on the technical characteristics of the system as well as on the way it is operated. It is considered, that losses up to $6-8 \%$ are normal, but the rates are up to $15-20 \%$ in the local grids.

It can be summed up:

\begin{tabular}{lll}
\hline Process & Amount & Energy \\
\hline Waste heat & $20-50 \%$ & $50-80 \%$ energy ultimate outcome \\
Generation & $30-35 \%$ & $15-28 \%$ energy ultimate outcome \\
Transmission & $6-8 \%$ & $13-25 \%$ energy ultimate outcome \\
\hline
\end{tabular}

It is seen that only 13-25\% of energy from fuel may be used by end customer. The technologies of energy conversion should be improved. The system has to be studied: burning device, concentration unit, optical fiber, photovoltaic device. Semiconductor with a long infrared area of radiation has to be selected. This will increase the efficiency of energetic system at least by $15-18 \%$.

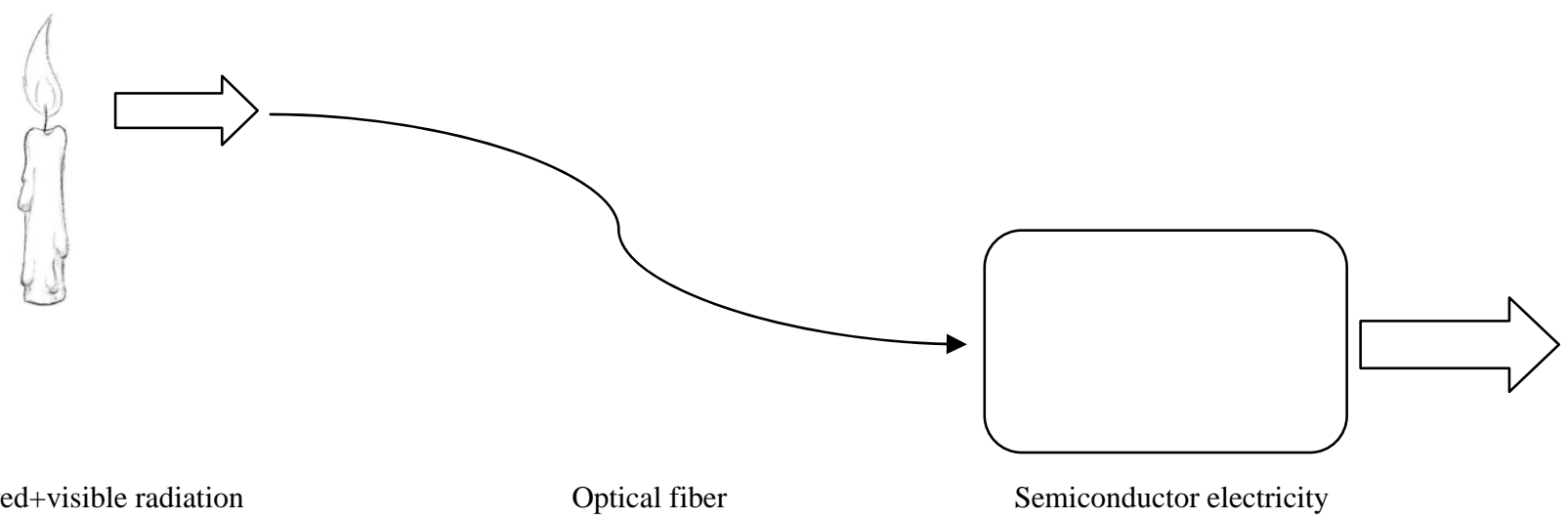




\section{References}

[1] Bernardi, M., Ferralis, N., Wan, J. H., Villalon, R., and Grossman, J. C. 2012. "Solar Energy Generation in Three Dimensions.” Energy Environ. Sci. 5: 6880-4.

[2] Yu, Y. L., Ferry, V. E., Alivisatos, A. P., and Cao, L. Y. 2012. "Dielectric Core-Shell Optical Antennas for Strong Solar Absorption Enhancement.” Nano Lett. 12 (7): 3674-81.

[3] Mavrokefalos, A., Han, S. E., Yerci, S., Branham, M. S., and Chen, G. 2012. "Efficient Light Trapping in Inverted Nanopyramid Thin Crystalline Silicon Membranes for Solar Cell Applications.” Nano Lett. 12 (6): 2792-6.

[4] Yablonovitch, E. 1987. "Statistical Ray Optics." J. Opt. Soc. Am. 72: 899-907.
[5] McCune, M., Zhang, W., and Deng, Y. L. 2012. "High Efficiency Dye-Sensitized Solar Cells Based on Three-Dimensional Multilayered ZnO Nanowire Arrays with 'Caterpillar-like' Structure.” Nano Lett. 12 (7): 3656-62.

[6] Kotelnikov, V. I., Barinov, A. V., and Ryazanova, E. A. 2015. “3D Solar Energy Concentration.” In Proceedings of the Conference on the 20th Anniversary of the Tuvinian Institute for Exploration of Natural Resources SB RAS, 244-6.

[7] Kotelnikov, V. I., and Ryazanova, E. A. 2015. “3D Solar Energy Conversion: Nano or Not.” In Proceedings of the 6th International Congress of Energy and Environment Engineering and Management (CIIEM15), 111-3. 